\title{
Basic Control Volume Finite Element Methods for Fluids and Solids
}


This page intentionally left blank 
IISc Research Monographs Series

\section{Basic Control Volume \\ Finite Element Methods \\ for Fluids and Solids}

Vaughan R Voller

University of Minnesota, USA

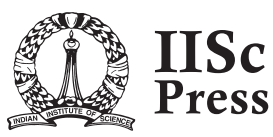


Published by

World Scientific Publishing Co. Pte. Ltd.

5 Toh Tuck Link, Singapore 596224

USA office: 27 Warren Street, Suite 401-402, Hackensack, NJ 07601

UK office: 57 Shelton Street, Covent Garden, London WC2H 9HE

\section{British Library Cataloguing-in-Publication Data}

A catalogue record for this book is available from the British Library.

\section{BASIC CONTROL VOLUME FINITE ELEMENT METHODS FOR FLUIDS AND SOLIDS \\ IISc Research Monographs Series - Vol. 1 \\ Copyright (C) 2009 by World Scientific Publishing Co. Pte. Ltd.}

All rights reserved. This book, or parts thereof, may not be reproduced in any form or by any means, electronic or mechanical, including photocopying, recording or any information storage and retrieval system now known or to be invented, without written permission from the Publisher.

For photocopying of material in this volume, please pay a copying fee through the Copyright Clearance Center, Inc., 222 Rosewood Drive, Danvers, MA 01923, USA. In this case permission to photocopy is not required from the publisher.

ISBN-13 978-981-283-498-0

ISBN-10 981-283-498-2

Printed in Singapore. 


\title{
Series Preface
}

\author{
World Scientific Publishing Company-Indian Institute of Science Collaboration
}

\begin{abstract}
World Scientific Publishing Company (WSPC) Singapore and Indian Institute of Science (IISc), Bangalore, will co-publish a series of prestigious lectures delivered during IISc's centenary year (2008-09), and a series of textbooks and monographs, by prominent scientists and engineers from IISc and other institutions.

This pioneering collaboration will contribute significantly in disseminating current Indian scientific advancement worldwide. In addition, the collaboration also proposes to bring the best scientific ideas and thoughts across the world in areas of priority to India through specially designed Indian editions.
\end{abstract}

\section{IISc Research Monographs Series}

The "IISc Research Monographs Series" will comprise state-of-the-art monographs written by experts in specific areas. They will include, but not be limited to, the authors' own research work.

K. Kesava Rao, Editor-in-Chief (kesava@chemeng.iisc.ernet.in)

H.R. Krishnamurthy (hrkrish@physics.iisc.ernet.in)

P. Vijay Kumar (vijay@ece.iisc.ernet.in)

Gadadhar Misra (gm@math.iisc.ernet.in)

S. Ramasesha (ramasesh@sscu.iisc.ernet.in)

Usha Vijayaraghavan (uvr@mcbl.iisc.ernet.in) 
This page intentionally left blank 
To my mentors, advisors, colleagues, and students 
This page intentionally left blank 


\section{Preface}

The advent of the digital computer in the middle of the last century initiated a rapid and continued growth in the development of computational tools for solving field problems. In the early days of these developments, researchers worked on a relatively small set of methods, applying them to a broad range of mechanics problems from stress and strain in solids through to fluid flow. As the field matured, however, distinct camps of researches based around methods and problems were formed. In extremely broad terms, the development of methods were split between those based on finite difference approaches and those based on finite element approaches; likewise applications were split between solids and fluids.

As the computational modeling field moved forward, other classes of solution methods were developed. Of particular note were control volume/finite volume methods. An immediate appeal of such methods was their obvious connection, through explicit discrete balance equations, to the physics of the problem at hand. Early control volume developments used finite difference methods to arrive at appropriate discrete equations. It was rapidly realized, however, that control volume solutions could also be constructed through the use of finite element technologies. Thus, control volume methods are viewed by some researchers as bridging between finite difference and finite element methods, with the ability to adopt and adapt the advantage of these methods while neglecting the drawbacks.

The Control volume methods that seem to obtain the maximum advantage of this hybrid view point are those based on finite element 
technologies, referred to as Control Volume Finite Element Methods (CVFEM). A notable feature of this class is the relative ease by which they can be applied to both solids and fluids problems. As such, the current research focused on solving multi-physics problems has spurred a significant interest in developing CVFEM solutions.

The central aim of this monograph is to introduce the basic and essential ingredients in control volume finite element methods. It is felt that this introduction will provide researchers with the critical background and base tools that will allow for more general application of CVFEM. Further, looking toward future multi-physics applications and trying to recapture the more comprehensive approach of the early days of computational mechanics, this monograph develops the basic constructions of CVFEM in the context of solving fundamental problems in both solids and fluids. This approach serves to fully highlight the generality and flexibility of CVFEM.

As with all efforts of this nature there is a host of people to thank. In general terms, I would first like to thank all of my mentors, advisors, colleagues, and students who have greatly contributed to my current understanding of computational mechanics. More explicit thanks need to go to the faculty of the Department of Mechanical Engineering at the Indian Institute of Science in Bangalore for providing motivation and support in this effort. I am also indebted to Mr Jim Hambleton who acted as a great sound board during the writing and provided a proof reading of the draft text.

V.R. Voller

Civil Engineering University of Minnesota 


\section{Contents}

Preface ix

1. Introduction 1

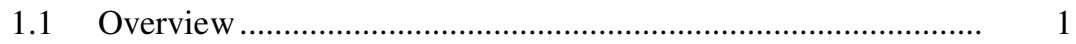

1.2 Objective and Philosophy......................................................... 2

1.3 The Basic Control Volume Concept .......................................... 3

1.4 Main Topics Covered ................................................................... 4

2. Governing Equations 7

2.1 The Euler Equations of Motion ................................................... 7

2.1.1 Conservation of mass ……............................................ 7

2.1.2 Conservation of linear momentum .................................... 8

2.1.3 Conservation of a scalar .................................................... 9

2.2 Specific Governing Equations .................................................... 9

2.2.1 Mass conservation in an incompressible flow ................. 10

2.2.2 Advection-diffusion of a scalar ....................................... 10

2.2.3 Stress and strain in an elastic solid ................................. 11

2.2.4 Plane stress ................................................................. 14

2.2.5 Plane strain .............................................................. 16

2.2.6 Relationship between plane stress and plane strain ......... 17

2.2.7 The Navier-Stokes equations ........................................... 18

2.2.8 The stream-function-vorticity formulation .................... 20

3. The Essential Ingredients in a Numerical Solution 22

3.1 The Basic Idea....................................................................... 22

3.2 The Discretization: Grid, Mesh, and Cloud.................................. 23

3.2.1 Grid...................................................................... 23

3.2.2 Mesh .................................................................... 23

3.2.3 Cloud .................................................................. 25

3.2.4 Discretizations for the Control Volume Finite Element Method 
3.3 The Element and the Interpolation Shape Functions .................... 25

3.4 Region of Support and Control Volume ........................................ 28

3.5 The Discrete Equation ................................................................ 29

4. Control Volume Finite Element Data Structure 32

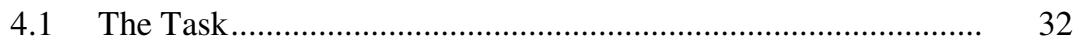

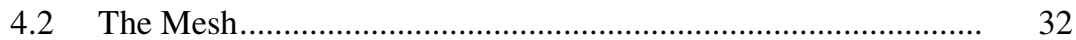

4.3 The Data Structure ….................................................................. 33

4.3.1 The region of support ……………………................... 33

4.3.2 The boundary............................................................... 35

4.4 The Discrete Equation ............................................................. 35

4.5 Summary ...................................................................... 38

5. Control Volume Finite Element Method (CVFEM)

Discretization and Solution $\quad 39$

5.1 The Approach ...................................................................... 39

5.2 Preliminary Calculations ............................................................ 41

5.3 Steady State Diffusion................................................................... 44

5.4 Steady State Advection-Diffusion ................................................. 46

5.5 Steady State Advection-Diffusion with Source Terms................. 49

5.5.1 Volume source terms ..................................................... 49

5.5.2 Source linearization ....................................................... 50

5.5.3 Line source ................................................................ 51

5.6 Coding Issues .................................................................... 52

5.7 Boundary Conditions ............................................................ 55

5.7.1 Face area calculations ................................................. 55

5.7.2 Convective condition ................................................... 57

5.7.3 Generalization of the convective boundary condition ..... 58

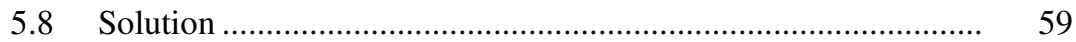

5.9 Handling Variable Diffusivity ................................................... 62

5.9.1 A conjugate problem .................................................... 62

5.9.2 Diffusivity a function of field variable .............................. 63

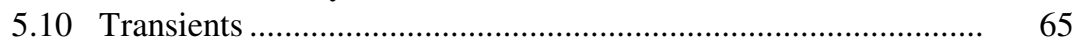

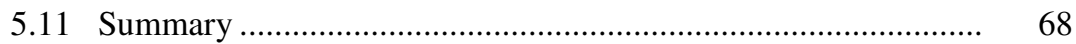

6. The Control Volume Finite Difference Method 69

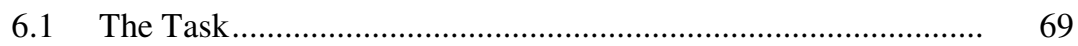

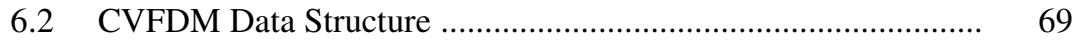

6.3 Coefficients and Sources ................................................................. 71

6.4 Boundary Conditions............................................................. 74

6.4.1 Insulated (no-flow) boundary ........................................ 74

6.4.2 Fixed value boundary ...................................................... 74 
7. Analytical and CVFEM Solutions of Advection-Diffusion Equations

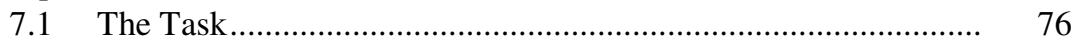

7.2 Choice of Test Problems ................................................................ 77

7.3 One-Dimensional Steady State Diffusion in a Finite Domain .... 80

7.4 One-Dimensional Transient Diffusion in a Semi-Infinite Domain

7.5 One-dimensional Transient Advection-Diffusion in a Semi-Infinite Domain.............................................................. 85

7.6 Steady State Diffusion in an Annulus ........................................ $\quad 87$

7.6.1 Constant diffusivity ...................................................... 87

7.6.2 Variable diffusivity and source term ................................ 90

7.7 Steady State Advection Diffusion in an Annulus ......................... 92

7.7.1 Constant diffusivity ......................................................... 92

7.7.2 Variable diffusivity......................................................... 94

7.8 Transient Diffusion from a Line Source ........................................ 95

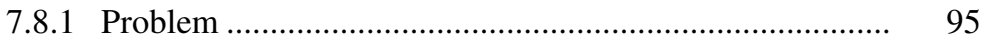

7.8.2 Unstructured mesh solutions …........................................ 97

7.8.3 Structured mesh solutions.................................................... 98

7.9 The Recharge Well Problem ....................................................... 100

8. A Plane Stress CVFEM Solution 106

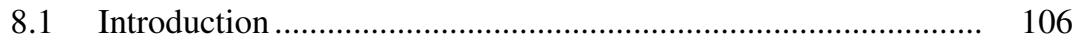

8.2 The Stress Concentration Problem ............................................. 106

8.3 CVFEM Displacement Solution.................................................. 107

8.3.1 The CVFEM discrete equations .................................... 109

8.3.2 Boundary conditions ........................................................ 111

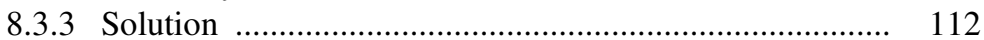

8.4 The Stress Solution ................................................................... 112

8.4.1 Stress in an element ..................................................... 115

8.4.2 Estimation of a nodal derivative ....................................... 115

8.4.3 Estimation of the nodal stress field ................................ 119

8.5 Summary ......................................................................... 120

9. CVFEM Stream function-Vorticity Solution for a

Lid Driven Cavity Flow $\quad 121$

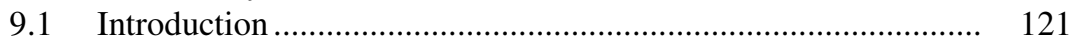

9.2 The Governing Equations...................................................... 122

9.3 The CVFEM Discretization of the Stream Function Equation.... 123 9.3.1 Diffusion contributions..................................................... 123 
9.3.2 Source terms

9.3.3 Boundary conditions........................................................ 126

9.4 The CVFEM Discretization of the Vorticity Equation ............... 126

9.4.1 Diffusion contributions.................................................. 126

9.4.2 The advection coefficients.............................................. 127

9.4.3 Boundary conditions...................................................... 128

9.5 Solution Steps ....................................................................... 129

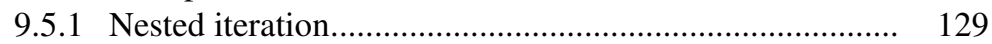

9.5.2 Calculating the nodal velocity field ................................. 130

9.6 Results .............................................................................. 132

10. Notes toward the Development of a 3-D CVFEM Code 138

10.1 The Tetrahedron Element ................................................... 138

10.2 Creating a Mesh of Tetrahedron Elements ............................... 139

10.3 Geometric Features of Tetrahedrons ……................................. 143

10.4 Volume Shape Functions ....................................................... 144

10.5 The Control Volume and Face .................................................. 146

10.6 Approximation of Face Fluxes ................................................ 148

10.6.1 Diffusive flux .......................................................... 148

10.6.2 Advective flux ............................................................. 149

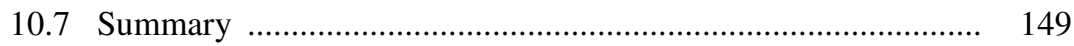

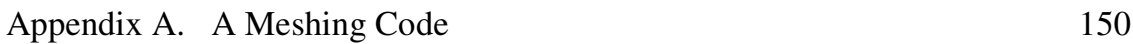

$\begin{array}{ll}\text { Appendix B. A CVFEM Code } & 158\end{array}$

$\begin{array}{ll}\text { Bibliography } & 167\end{array}$

$\begin{array}{ll}\text { Index } & 169\end{array}$ 